\title{
PARALLELOHEDRA: A RETROSPECTIVE AND NEW RESULTS
}

\author{
N. P. DOLBILIN
}

\begin{abstract}
Parallelohedra are polyhedra that partition Euclidean space with parallel copies. This class of polyhedra has applications both in mathematics and in the natural sciences. An important subclass of parallelohedra is comprised of the so-called Voronoŭ parallelohedra, which are Dirichlet-Voronoŭ domains for integer lattices. More than a century ago Voronor stated the conjecture that every parallelohedron is affinely equivalent to some Voronor parallelohedron. Although considerable progress has been made, this conjecture has not been proved in full. This paper contains a survey of a number of classical theorems in the theory of parallelohedra, together with some new results related to Voronou's conjecture.
\end{abstract}

\section{GENERAL INFORMATION ABOUt PARALlELOHEDRA}

Parallelohedra have applications in mathematics and then natural sciences. Multidimensional parallelohedra are applied to the geometry of numbers, combinatorial geometry and coding theory. A three-dimensional parallelohedron gives a model of the fundamental cell of a crystal and plays an important role in geometric crystallography, structural chemistry and solid-state physics. This class of polyhedra is also interesting in its own right. On the one hand, it is completely described by simple conditions (the Minkowski-Venkov criterion), and on the other hand, as we shall see, this class is very numerous and many natural questions on combinatorics of parallelohedra remain unsolved.

Both the notion and the term parallelohedron were introduced by the prominent crystallographer Fëdorov [1 in 1885. The term is related to the fact that, for a polyhedron partitioning space with parallel copies, every face 1 (in multi-dimensional space, hyperface) has an equal and opposite parallel face of codimension 1.

Recall that a partition of space into polyhedra is defined as an arrangement of polyhedra $\left\{P_{1}, P_{2}, \ldots\right\}$ in $\mathbb{E}^{d}$ such that

(1) pairwise they do not overlap, that is, they have no common interior points:

$$
\operatorname{Int}\left(P_{i}\right) \cap \operatorname{Int}\left(P_{j}\right)=\varnothing ;
$$

(2) their union forms a covering of space:

$$
\bigcup_{i} P_{i}=\mathbb{E}^{d}
$$

The polyhedra $P_{i}$ that form a partition are also called cells. We shall only consider partitions into convex cells and say that a partition is normal if any two of its cells which intersect (over a boundary) intersect over an entire face of some dimension.

2010 Mathematics Subject Classification. Primary 52B20; Secondary 52B11, 52B12.

Key words and phrases. Parallelohedron, Minkowski-Venkov criterion for parallelohedra, Voronoı̌'s conjecture on parallelohedra, standard face, index of a face.

This research was supported by the Government of the Russian Federation (grant no. 11.G34.31.0053) and the Russian Foundation for Basic Research (grant no. 11-01-00633-a).

${ }^{1}$ From the Greek hédron meaning base or face. 

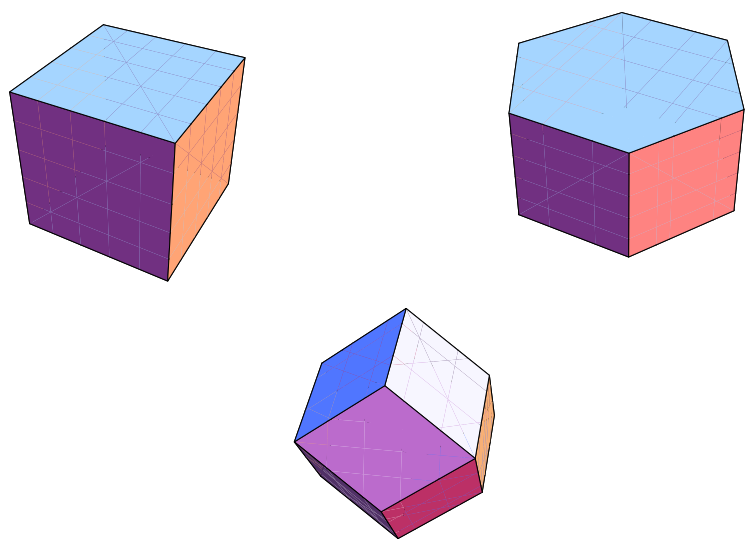

Figure 1. Cube; hexagonal prism; rhombic dodecahedron

A parallelohedron of dimension $d$ is defined to be a closed convex Euclidean $d$-dimensional polyhedron $P \subset \mathbb{E}^{d}$ which, together with its parallel copies, gives a normal partition of the space $\mathbb{E}^{d}$.

If a partition $T$ into parallelohedra is normal, it is uniquely defined by the parallelohedron $P$ itself and its position in space. Indeed, in the partition $T$, along with the parallelohedron $P$, consider all the parallelohedra $P_{1}, \ldots, P_{m}$ adjacent to it over all $m$ hyperfaces, and the vectors $\boldsymbol{t}_{1}, \ldots, \boldsymbol{t}_{m}$ such that $P+\boldsymbol{t}_{i}=P_{i}, i=1, \ldots, m$. Any other parallelohedron $P^{\prime} \in T$ is situated parallel to the parallelohedron $P$, and therefore each translation $\boldsymbol{t}_{i}$ also takes $P^{\prime}$ to a parallelohedron $P^{\prime}+\boldsymbol{t}_{i}$ of the partition $T$ adjacent to $P^{\prime}$ over the corresponding hyperface. Thus, the translation $\boldsymbol{t}_{i}$ leaves the partition $T$ invariant: $T+\boldsymbol{t}_{i}=T$.

Thus, the group $\Lambda$ generated by the translations $\boldsymbol{t}_{i}$ is a translation subgroup of the full symmetry group of the partition $T$. The group $\Lambda$ acts transitively on the set of parallelohedra of the partition $T$. Obviously,

$$
\Lambda=\left\{\sum_{i}^{m} n_{i} \boldsymbol{t}_{i}, n_{i} \in \mathbb{Z}\right\} .
$$

It is known (see 5]) that the group $\Lambda$ is an integer lattice of rank $d$, that is, there exists a basis $\mathcal{E}=\left\{\boldsymbol{e}_{1}, \ldots, \boldsymbol{e}_{d}\right\}$ in $\Lambda$ consisting of exactly $d$ vectors such that $\Lambda=\left\{\sum_{i}^{d} n_{i} \boldsymbol{e}_{i}\right.$, $\left.n_{i} \in \mathbb{Z}\right\}$ (see also Problem 1 in $\S 6$ of this paper).

Thus, by normality the partition $T$ into parallelohedra is a lattice partition in the sense that $T=P+\Lambda$ for some lattice $\Lambda$. This remark is equivalent to the fact, important in crystallography, that a parallelohedron is a fundamental domain for the discrete group of parallel translations of a lattice.

Fëdorov found all five combinatorial types of three-dimensional parallelohedra (see Figures 1 and 2). These are the cube, hexagonal prism, two dodecahedra (the rhombic dodecahedron and elongated dodecahedron), and one more parallelohedron with 14 faces. This is the so-called truncated octahedron. Recall that two polyhedra are combinatorially equivalent if there exists a bijection between the sets of their faces of all dimensions, partially ordered by inclusion, that preserves the dimensions and incidence of the corresponding faces.

When deriving the three-dimensional parallelohedra, Fëdorov used the fact that parallelohedra are centrally symmetric polyhedra. He thought this was self-evident, because 

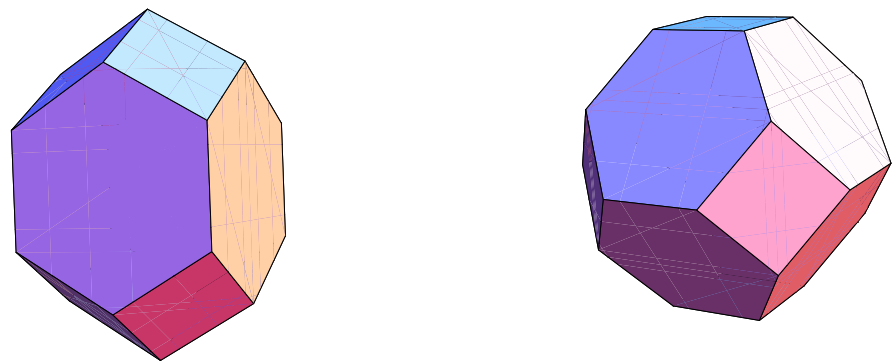

FIGURE 2. Elongated dodecahedron; truncated octahedron

a partition is a lattice by nature. However, as is shown by examples, if we do not require that the cell of a partition into parallel copies be convex, then, although the lattice nature of the partition is preserved, the cell does not have to be centrally symmetric. In order to prove this "self-evident" fact, Minkowski discovered and proved his amazing theorem on convex polyhedra (see [2]), from which he immediately obtained the central symmetry of a parallelohedron.

In a partition consider two adjacent parallelohedra $P$ and $P_{i}$ with a common hyperface $F_{i}$ and the segment $O O_{i}$ connecting their centres. The fact that the parallelohedron is a centrally symmetric polyhedron implies that the symmetry with respect to the midpoint $O_{i}^{\prime}$ of the segment $O O_{i}$ interchanges the parallelohedra $P$ and $P_{i}$ and leaves invariant the common hyperface $F_{i}$. The vectors $\boldsymbol{t}_{i}=\overrightarrow{O O_{i}}, i=1,2, \ldots, m$, are called facet vectors (facet is another term for a hyperface).

Minkowski found the upper estimate $2\left(2^{d}-1\right)$ for the number of hyperfaces of a $d$ dimensional parallelohedron (Theorem 1.4). This estimate is exact: in a space of any dimension there exist so-called primitive parallelohedra with exactly $2\left(2^{d}-1\right)$ hyperfaces. Recall that a parallelohedron is said to be primitive if at every vertex the minimum number of cells for the given dimension $d$ meet, namely $d+1$.

At the same time there exist a huge number of nonprimitive parallelohedra for which the estimate $2\left(2^{d}-1\right)$ is strictly greater than the number of hyperfaces they have. In this paper (§3) we derive Minkowski's estimate from the index theorem (Theorem 13.1), which was proved by the author in [21. The index theorem not only implies Minkowski's estimate but also gives an explanation of why there is a difference between this estimate and the number of hyperfaces that nonprimitive parallelohedra have.

Note that Minkowski's theorem on necessary conditions was supplemented by the converse theorem on sufficient conditions (Venkov [7]; see also [9], [16], and [17]). The Minkowski-Venkov criterion shows that parallelohedra have so-called belts (see Definition 1) of length either 4 or 6 .

If all the belts of some parallelohedron have length 4 , then it is a parallelepiped. However, allowing parallelohedra to have belts of length 6 changes the picture fundamentally. Despite the simplicity of the criterion, the realm of parallelohedra is striking in its numerousness and diversity. For example, in 4-dimensional space there are in total 52 combinatorial types of parallelohedra (Delone [4, Aleksandrov and Shtogrin 12]). In 5-dimensional space all 222 combinatorial types of primitive parallelohedra have been determined (Ryshkov and Baranovskiı̌ [10, Engel and Grishukhin [18]). Engel's calculations show that combinatorial types of 5 -dimensional parallelohedra are numbered in thousands [13, and of 6-dimensional in millions. 
In 1908 Voronoŭ proved a deep theorem that every primitive parallelohedron is affinely equivalent to some parallelohedron that is a Dirichlet-Voronor domain for an integer lattice. Such parallelohedra are now universally called Voronő parallelohedra.

Voronol's strategy was to reduce the problem of enumerating the types of all parallelohedra to enumerating the types of Voronol parallelohedra. Voronoŭ stated the conjecture that every parallelohedron (not just the primitive ones, as he proved) is affinely equivalent to some Voronol parallelohedron. Then he constructed the theory of Voronol parallelohedra, which in fact contains a method for enumerating all the combinatorial types of $d$-dimensional Voronoı̆ parallelohedra for a given dimension $d$. As for Voronol's conjecture, it remains unproved, even though Zhitomirskil strengthened Voronoü's theorem substantially [6], and there have been other results obtained in this direction (see, for example, [14, [19], 25]).

Note that Voronol's theorem is an existence theorem - there exists at least one Voronol parallelohedron in the affine class of every primitive parallelohedron. In this paper a uniqueness theorem for the parallelohedron is proved under the most general conditions on the parallelohedron for which this theorem remains true. In fact, the first uniqueness theorem appeared in [11, where it was proved that for every primitive parallelohedron, the Voronol parallelohedron in its affine class is unique up to similarity. In particular, this theorem implies that in a given domain of common Voronor type, two Voronoŭ parallelohedra are either similar to each other or are affinely nonequivalent. This caused some surprise, because it is well known that, by contrast, the Delone partitions dual to Voronol partitions within a given domain of type all, without exception, belong to the same affine class.

In our paper we present a retrospective view of the important results in the theory of parallelohedra that relate directly to the further contents of the paper. In what follows, the class of standard faces is distinguished in the lattice of faces of a parallelohedron. We have succeeded in reformulating and reproving several classical theorems on parallelohedra in terms of standard faces, and also obtained a number of new results. For example, we derive the upper estimate for the number of hyperfaces from the index theorem. In conclusion we give a purely geometric proof of the theorem that the Voronor parallelohedron in the affine class of a given parallelohedron is unique, under the most general conditions on the polyhedron. Note that the uniqueness theorem does not hold under weaker conditions.

\section{$\S$ 1. Minkowski's THEOREMS ON PARALLELOHEDRA}

Theorem 1.1 (Minkowski [2]). If $P$ is a d-dimensional parallelohedron, then

(1) $P$ is centrally symmetric;

(2) all its hyperfaces are centrally symmetric;

(3) the projection of $P$ along each of its $(d-2)$-faces onto the complementary 2-plane is either a parallelogram or a centrally symmetric hexagon.

As we have already said, the main thing is to prove condition (1). This condition follows from the celebrated Minkowski theorem on convex polyhedra.

Let $P$ be a bounded convex polyhedron with $m$ hyperfaces $F_{1}, \ldots, F_{m}$. Let $\boldsymbol{n}_{i}$ denote the outward normal to the hyperface $F_{i}$, and $s_{i}$ its $(d-1)$-dimensional volume. The set of vectors $\mathcal{N}:=\left\{s_{1} \boldsymbol{n}_{1}, \ldots, s_{m} \boldsymbol{n}_{m}\right\}$ is called the hedgehog of the polyhedron $P$. It is easy to show that the hedgehog of every bounded polyhedron satisfies the following two conditions:

(1) $\operatorname{dim} \operatorname{span}(\mathcal{N})=d$;

(2) $\sum_{i=1}^{m} s_{i} \boldsymbol{n}_{i}=0$. 
Theorem 1.2 (Minkowski [2], 1897; see also [8]). Suppose that $\mathcal{N}=\left\{\boldsymbol{N}_{1}, \ldots, \boldsymbol{N}_{m}\right\}$ is a set of vectors in d-dimensional space satisfying the conditions

(1) $\operatorname{dim} \operatorname{span}(\mathcal{N})=d$;

(2) $\sum_{i=1}^{m} \boldsymbol{N}_{i}=0$.

Then there exists a convex d-polyhedron $P$ with $m$ hyperfaces for which the set $\mathcal{N}$ is the hedgehog.

Moreover, the polyhedron $P$ is determined by its hedgehog $\mathcal{N}$ uniquely up to a parallel translation.

Proving the uniqueness in this theorem is quite difficult. But it is the fact of uniqueness that implies many useful consequences, in particular the following.

Corollary 1.1. A convex polyhedron, each of whose hyperfaces has a hyperface parallel to it with the same $(d-1)$-dimensional volume, is centrally symmetric.

It is easy to see that the hedgehog $\mathcal{N}$ of a polyhedron $P$ satisfying the hypotheses of Corollary 1.1 is centrally symmetric. Next, a symmetry (with centre at an arbitrary point of space) takes the polyhedron $P$ to a polyhedron $P^{\prime}$ symmetric to it. The hedgehog of the polyhedron $P^{\prime}$ is symmetric to the hedgehog $\mathcal{N}$. But since the hedgehog $\mathcal{N}$ of the polyhedron $P$ is centrally symmetric, the polyhedra $P$ and $P^{\prime}$ have the same hedgehogs. Hence the polyhedron $P^{\prime}$, on the one hand, is symmetric to the polyhedron $P$. On the other hand, these polyhedra, by Minkowski's theorem, can be superposed by a parallel translation. This immediately implies that the polyhedron $P$ has a centre of symmetry.

Since the set of all hyperfaces of a parallelohedron is divided into pairs of parallel hyperfaces, equal to each other, the parallelohedron is centrally symmetric (see part (1) of Theorem 1.1).

Theorem 1.3 (Aleksandrov, $d=3$; Shephard, $d>3)$. A convex $d$-polyhedron $(d>2)$, all of whose hyperfaces are centrally symmetric, is also centrally symmetric.

By this theorem, properties (1) and (2) in Theorem 1.1 are not independent. Property (1) follows from property (2). Nevertheless, property (1) is always included in the statement of the theorem because in all the proofs of Theorem 1.1 which are known at present (1) is proved first, and (2) is derived using (1). Here we shall give a new, quite simple proof of the Aleksandrov-Shephard theorem.

But before we do this we introduce the notion of a belt for $d$-polyhedra with centrally symmetric hyperfaces. Consider a $(d-2)$-dimensional face $F^{d-2} \subset F$. The hyperface $F\left(=F_{1}\right)$ contains the face $F_{2}^{d-2}$ symmetric to $F^{d-2}\left(=F_{1}^{d-2}\right)$ with respect to the centre of the hyperface $F_{1}$. Now let $F_{2}^{d-2}$ be the common $(d-2)$-face for the hyperfaces $F_{1}$ and $F_{2}: F_{2}^{d-2}=F_{1} \cap F_{2}$. Also let the face $F_{3}^{d-2} \subset F_{2}$ be symmetric to the face $F_{2}^{d-2}$ with respect to the centre of the hyperface $F_{2}$, and so on.

In this fashion we can construct a closed chain of some number $p$ of hyperfaces $F_{1}(=F), F_{2}, \ldots, F_{p}, F_{p+1}=F_{1}$ of the polyhedron such that every pair of consecutive hyperfaces $F_{i-1}, F_{i}$ has a common face $F_{i}^{d-2}=F_{i-1} \cap F_{i}$ for $i=2, \ldots, p+1$, and $F_{1}^{d-2}=F_{p} \cap F_{1}$ for $i=1$.

Definition 1. The chain of hyperfaces described above is called the belt $B=B\left(F^{d-2}\right)$ corresponding to the face $F^{d-2}$.

All the intermediate $(d-2)$-faces $F_{1}^{d-2}, F_{2}^{d-2}, \ldots, F_{p}^{d-2}$ in the belt are connected by the symmetries with respect to the centres of hyperfaces: the face $F_{i}^{d-2}, i>1$, is obtained from the face $F_{1}^{d-2}$ by the composition of the symmetries with respect to the centres of the hyperfaces $F_{1}, F_{2}, \ldots, F_{i-1}$. 
Hence all the faces $F_{i}^{d-2}$ are congruent to one another and lie in pairwise parallel $(d-2)$-planes aff $\left(F_{i}^{d-2}\right)$, which are parallel to the hyperplane aff $(F)$. Thus, if we project the polyhedron along the $(d-2)$-plane aff $\left(F^{d-2}\right)$ onto the orthogonal complement $\Pi$, then in the 2-plane $\Pi$ we obtain a $p$-gon whose sides are the projections of the hyperfaces of the belt $B$ and whose vertices are the projections of the faces $F_{i}^{d-2}, i=1,2, \ldots, p$.

Proof of Theorem 1.3. We claim that for any hyperface $F$ of a polyhedron $P$ with centrally symmetric hyperfaces there is an equal and parallel opposite hyperface $F^{\prime}$. By Minkowski's theorem this will imply the central symmetry of the $d$-dimensional polyhedron (see Corollary 1.1).

Take a hyperface $F$, a $(d-2)$-subface $F^{d-2} \subset F$, and the corresponding belt $B=$ $B\left(F^{d-2}\right)$.

Consider the hyperplane of support $H$ containing the hyperface $F=H \cap P$, and the other parallel hyperplane of support $H^{\prime}$. We claim that the hyperplane of support $H^{\prime}$ also contains a hyperface of the polyhedron $P$. Let $\pi: P \rightarrow \Pi$ be the projection of the polyhedron $P$ along aff $\left(F^{d-2}\right)$ onto the orthogonal 2-complement $\Pi$.

Obviously, $h:=\pi(H)$ and $h^{\prime}:=\pi\left(H^{\prime}\right)$ are straight lines of support for the polygon $M:=\pi(P)$. Furthermore, the straight line of support $h$ contains a side of the polygon $M$, while the straight line $h^{\prime}$ contains at least one vertex $v$ of the polygon. This means that the hyperplane $H^{\prime}$ contains the pre-image $\pi^{-1}(v)$ :

$$
H^{\prime} \supset \pi^{-1}(v)=\operatorname{aff}\left(F_{k}^{d-2}\right) \supset F_{k}^{d-2} .
$$

Thus, the hyperplane of support $H^{\prime}$ parallel to the hyperplane $H \supset F$ must contain, if not a hyperface, then at least some $(d-2)$-face $F_{k}^{d-2}$ parallel to the face $F^{d-2} \subset F$.

Consider another $(d-2)$-face $F^{\prime} d-2$ in the hyperface $F$ that is not parallel to the $(d-2)$-face $F^{d-2}$. We can repeat the same arguments for it as for $F^{d-2}$. Since the hyperplane of support $H^{\prime}$ parallel to the plane $H$ of the hyperface $F$, is unique, $H^{\prime}$ contains a $(d-2)$-face $F_{l}^{\prime} d-2$ parallel to the face $F^{\prime} d-2$. Thus, the plane of support $H^{\prime}$ contains at least two faces of codimension 2 that are not parallel to each other, and therefore it contains the whole hyperface $F^{\prime}$ that is parallel to the hyperface $F$.

By conducting the same arguments for any face $F^{\prime \prime} d-2 \subset F$ of codimension 2 we obtain a face in the hyperface $F^{\prime}$ parallel to it. Since hyperfaces are centrally symmetric, for every pair of mutually symmetric faces of codimension 2 contained in the hyperface $F$ there is a parallel pair of faces congruent to them in the hyperface $F^{\prime}$. Thus, we have two $(d-1)$-dimensional polyhedra $F$ and $F^{\prime}$ that lie in parallel $(d-1)$-planes. Moreover, for every pair of mutually symmetric $(d-2)$-faces of the polyhedron $F$, in the polyhedron $F^{\prime}$ there is a parallel pair of congruent $(d-2)$-faces that are also symmetric to each other with respect to the centre of the polyhedron $F^{\prime}$. Applying Minkowski's theorem to the $(d-1)$-polyhedra $F$ and $F^{\prime}$ we see that they are congruent and situated in parallel.

Thus, we have proved that in the $d$-polyhedron $P$ every hyperface $F$ has a hyperface $F^{\prime}$ congruent to it and situated in parallel. The hedgehog of such a polyhedron is centrally symmetric. By Minkowski's theorem the $d$-polyhedron $P$ is also centrally symmetric.

We present another important theorem of Minkowski's.

Theorem 1.4. The number $f_{d-1}$ of hyperfaces in a d-parallelohedron satisfies the inequality

$$
f_{d-1} \leq 2\left(2^{d}-1\right)
$$

Recall that the estimate is best-possible. Note also that among $d$-parallelohedra, it is the parallelepiped one that has the minimal number of hyperfaces: $2 d$. 

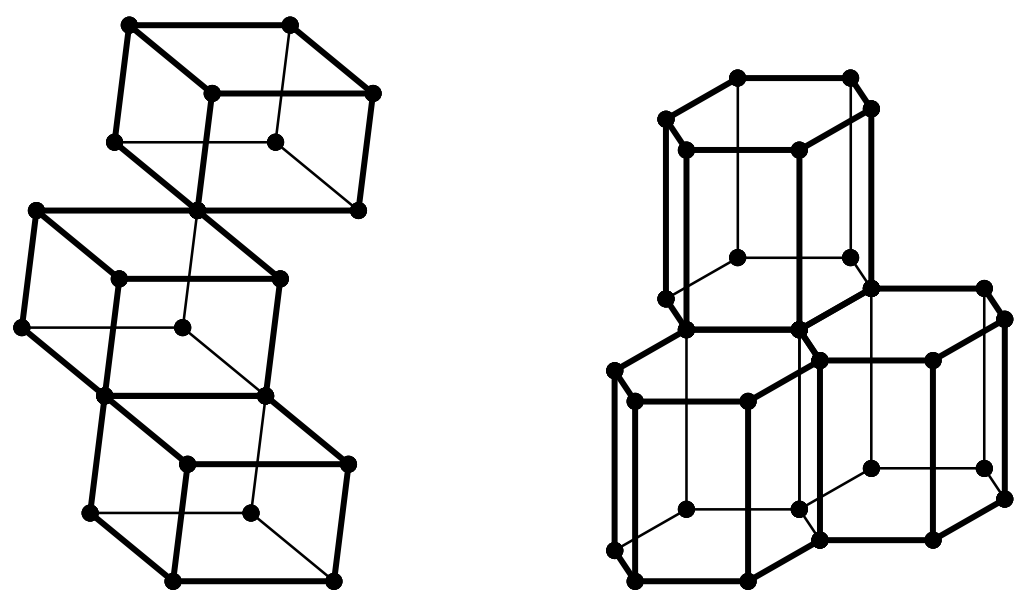

Figure 3. Examples of standard faces

\section{$\S 2$. Standard FaCES}

The definition of a standard face of a parallelohedron was introduced by the author; this subsequently proved to be useful (see [21, and also [20]). In addition, this definition is suitable for any normal partition into polyhedra. Let $T$ be a normal (locally finite) partition of space into convex polyhedra, and let $P, P^{\prime} \in T$ be cells that have nonempty intersection: $P \cap P^{\prime} \neq \varnothing$. Since $T$ is normal, the intersection $P \cap P^{\prime}$ is the face $F^{i}$ common to these cells, with some dimension $i, 0 \leq i \leq d-1$. However, not every face of the partition can be represented as the intersection of two cells.

Definition 2. A face $F^{i}$ in a partition $T$ is said to be standard if it can be represented as the intersection $F^{i}=P \cap P^{\prime}$ of two cells $P$ and $P^{\prime}$ in $T$.

Obviously, in a normal partition every hyperface is standard and every face of the partition is contained in some standard face.

We will now restrict ourselves to parallelohedra. Since a given parallelohedron determines the partition uniquely, in the case of a partition into parallelohedra we can speak not of a standard face of the partition but of a standard face of the parallelohedron itself. There are parallelohedra such that not every face is standard. In fact, if a parallelohedron is primitive, then none of its faces, apart from the hyperfaces, are standard [21. A face of codimension 2 is standard if and only if the corresponding belt (see Definition 1 ) has length 4. For faces of other dimensions, conditions for a face to be standard in terms of the parallelohedron itself are unknown.

We consider several examples. In a partition of the plane into hexagons, any nonempty intersection of two cells must be a common side, but not a vertex.

In a $d$-parallelepiped, all faces of any dimension $i, 0 \leq i \leq d-1$, are standard (see Figure 3).

In a three-dimensional hexagonal prism, all the 2-faces and the 12 edges lying in the bases are standard. But all of its 6 lateral edges and its 12 vertices are not standard faces.

Recall some facts about standard faces in [21]:

1. A standard face of a parallelohedron is centrally symmetric, and the centre of symmetry of a standard face is a point of the set $\frac{1}{2}(\Lambda \backslash 2 \Lambda)$.

2. Conversely, any point in $\frac{1}{2}(\Lambda \backslash 2 \Lambda)$ is the centre of symmetry of a standard face $F$ of a parallelohedron in the partition $T$. 
Note that being centrally symmetric is not a sufficient condition for a face to be standard. For example, every edge is centrally symmetric, but not every edge is a standard face. For example, as mentioned above, a lateral edge of a hexagonal prism is not a standard face.

Recall that the set of all parallelohedra of a partition meeting at a face $F$ of the partition is called the star $\operatorname{St}(F)$ of the face $F$. Suppose that a face $F$ of a partition is not standard, and let parallelohedra $P$ and $P^{\prime}$ belong to the $\operatorname{star} \operatorname{St}(F)$. Then their common face $F^{\prime}=P \cap P^{\prime}$ is a standard face, which contains the nonstandard face $F$ as a proper subface.

This implies that if the codimension of a face $F$ is equal to 2 and the face $F$ is not standard, then every two parallelohedra in $\operatorname{St}(F)$ must have a common hyperface. But this is possible if and only if exactly three parallelohedra meet at the $(d-2)$-face.

If, however, a $(d-2)$-face $F$ is standard, then $\operatorname{St}(F)$ contains at least 4 parallelohedra. Also, since any two parallelohedra $P$ and $P^{\prime}$ in $\operatorname{St}(F)$ that intersect only in the face $F$ $\left(P \cap P^{\prime}=F\right)$ must be mutually symmetric with respect to the centre of symmetry of the face $F$, it follows that there are at most four parallelohedra in $\operatorname{St}(F)$.

Definition 3. The degree $\operatorname{deg}(F)$ of an arbitrary face $F$ in a partition $T$ is the number of parallelohedra in $\operatorname{St}(F)$.

What has just been said implies the following.

Corollary 2.1. The degree of a $(d-2)$-face of a parallelohedron is equal to either 3 or 4. Moreover, $\operatorname{deg}\left(F^{d-2}\right)=4$ if and only if $F^{d-2}$ is standard.

This assertion is equivalent to property (3) in Minkowski's Theorem 1.1 (see [21]). Thus, property (3) of Theorem 1.1 is derived from the properties of a standard $(d-2)$ face of a parallelohedron.

\section{$\S$ 3. The INDEX THEOREM AND AN ESTIMATE FOR THE NUMBER OF HYPERFACES}

We are given a partition into parallelohedra, and $F$ is an arbitrary face of the partition.

Definition 4. The index of a face $F$ is the number $\nu(F)$ inverse to its degree: $\nu(F):=$ $\frac{1}{\operatorname{deg}(F)}$.

Clearly, the index of any hyperface is equal to $\frac{1}{2}$. The index of a $(d-2)$-face of a parallelohedron is equal to $\frac{1}{4}$ if the face is standard, and to $\frac{1}{3}$ otherwise. Next, since for any $(d-i)$-dimensional face $F^{d-i}$ its degree satisfies $\operatorname{deg}\left(F^{d-i}\right) \leq 2^{i}$ (see [24]), we have $\nu\left(F^{d-i}\right) \geq \frac{1}{2^{i}}$.

The set of all standard faces of a parallelohedron $P$ is denoted by $\mathscr{S}$.

Theorem 3.1 (Index Theorem, [21]). Given an arbitrary parallelohedron the sum of indices of all its standard faces is equal to $2^{d}-1$, that is,

$$
\sum_{F \in \mathscr{S}} \nu(F)=2^{d}-1
$$

The index theorem immediately implies Minkowski's upper estimate (Theorem 1.4) for the number of hyperfaces in a $d$-dimensional parallelohedron. We introduce the following notation:

$\mathscr{S}^{i}$ is the set of all standard $i$-faces in $P$,

$\mathscr{S}^{\prime}$ is the set of all standard $i$-faces in a parallelohedron $P$ for $i \leq d-2$.

Obviously, $\mathscr{S}=\mathscr{S}^{d-1} \cup \mathscr{S}^{\prime}$ and $\left|\mathscr{S}^{d-1}\right|=f_{d-1}$. Relation (1) implies

$$
2^{d}-1=\sum_{F \in \mathscr{S}} \nu(F)=\sum_{F \in \mathscr{S}^{d-1}} \nu(F)+\sum_{F \in \mathscr{S}^{\prime}} \nu(F) \geq \frac{1}{2} f_{d-1},
$$


whence we obtain Minkowski's estimate:

$$
f_{d-1} \leq 2\left(2^{d}-1\right)
$$

Since in a primitive parallelohedron only hyperfaces are standard faces, that is, $\mathscr{S}^{\prime}=$ $\varnothing$, for primitive parallelohedra equality in (3) is attained. Note that starting from $d=4$ there are nonprimitive $d$-parallelohedra with $2\left(2^{d}-1\right)$ hyperfaces (oral communication from Shtogrin). It follows from relation (11) that such parallelohedra have no other standard faces, except for hyperfaces.

However, if $\mathscr{S}^{\prime} \neq \varnothing$ for a parallelohedron, then by (2) the number of hyperfaces diminishes:

$$
f_{d-1}=2\left(2^{d}-1\right)-2 \sum_{F \in \mathscr{S}^{\prime}} \nu(F)
$$

\section{$\S$ 4. VORONOĬ'S CONJECTURE}

Let $\Lambda \subset \mathbb{E}^{d}$ be an integer lattice of rank $d$, let $\mathscr{V}(\Lambda)$ be the Voronou partition of the space $\mathbb{E}^{d}$ for the lattice $\Lambda$, and let $V(\lambda)$ be the Voronor domain for a point $\lambda \in \Lambda$. First, the Voronor partition is normal. Second, since the lattice is the orbit of a point with respect to the translational group of the lattice, the Voronol domains for points of the lattice are congruent and are taken to one another by parallel translations. Thus, the Voronor cell for a point of the lattice is a parallelohedron, which is called a Vorono $\breve{\imath}$ parallelohedron.

Not every parallelohedron is a Voronou parallelohedron. For example, for $d=2$ two-dimensional parallelohedra (parallelograms and centrally symmetric hexagons) are Voronoŭ parallelohedra if and only if they are inscribed in a circle.

Consider the star of a face $\operatorname{St}\left(F^{k}\right)$. We say that a parallelohedron $P$ is primitive at the face $F^{k} \subset P$ if $\operatorname{deg}\left(F^{k}\right)=d+1-k$.

It is easy to verify that if a parallelohedron is primitive at a face $F^{k}$, then it is primitive at any of its overfaces $F^{m} \supset F^{k}, m>k$, that is, $\operatorname{deg}\left(F^{m}\right)=d+1-m$.

Hence a primitive parallelohedron is primitive at any of its faces. Generally speaking, this property does not extend in the reverse direction: for example, a rhombic dodecahedron is primitive at every edge, but is not primitive at some vertices.

Theorem 4.1 (Voronor [3], 1908). Any primitive parallelohedron is affinely equivalent to some Voronoŭ parallelohedron.

Voronor also conjectured that any parallelohedron is affinely equivalent to some Vorono $\breve{~}$ parallelohedron (Voronoù's conjecture).

Theorem 4.2 (Zhitomirskiu [ $[$ ]). A parallelohedron that is primitive at every $(d-2)$-face is affinely equivalent to some Voronoŭ parallelohedron.

Zhitomirskilı's theorem can be reformulated in terms of standard faces: if a parallelohedron has no standard faces of codimension 2 , then it is affinely equivalent to some Voronol̆ parallelohedron.

Following Zhitomirskiıl's theorem, Voronol's conjecture only remains to be proved for those parallelohedra that have standard $(d-2)$-dimensional faces. Ordin [19] has an interesting result which further strengthens Zhitomirski1̌'s result, and very recently another result was obtained that also strengthens Zhitomirskiı̌'s theorem (see [25]).

Let $\mathscr{F}_{s}^{d-2}$ denote the union of all closed standard faces of codimension 2 of a parallelohedron $P$.

Theorem 4.3 (Gavrilyuk, Garber and Magazinov [25]). Let $P$ be a parallelohedron such that $Q:=\partial P \backslash \mathscr{F}_{s}^{d-2}$ is a simply connected $(d-1)$-dimensional manifold. Then $P$ is affinely equivalent to some Voronő parallelohedron. 
Standing somewhat away from the Voronou-Zhitomirskiu direction there is the wellknown result of Erdahl, who proved Voronol's conjecture for parallelohedra that are zonotopes, that is, polyhedra that can be represented as the Minkowski sum of finitely many segments [14]. For $d \leq 4$ all parallelohedra are zonotopes. However, starting with $d=5$ the class of parallelohedra-zonotopes no longer contains the entire class of all parallelohedra that have no standard $(d-2)$-faces (the Zhitomirskiu case), while on the other hand this class contains a significant part of the parallelohedra with standard $(d-2)$-faces.

Along with the partition into Voronol parallelohedra, there is the Delone partition constructed for the same lattice. In fact, the theory of Voronol and Delone partitions dual to each other was developed by Delone for quite arbitrary discrete sets, the so-called $(r, R)$-systems (nowadays called Delone sets). For example, Theorem 4.4 below holds for arbitrary Delone sets rather than just lattices.

Let $\Lambda \subset \mathbb{E}^{d}$ be a point lattice; a $d$-polyhedron $D$ is called a Delone polyhedron for this lattice $\Lambda$ if it satisfies the following two conditions:

(1) $\operatorname{Vert}(D)=\Lambda \cap S^{d-1}$, where $S^{d-1}$ is some $(d-1)$-sphere;

(2) there are no points in $\Lambda$ inside the sphere $S^{d-1}$.

Theorem 4.4 (Vorono $[3$, Delone [5]). The set of all Delone polyhedra possible for a given lattice is a normal partition, the so-called Delone partition 2 of the space $\mathbb{E}^{d}$. The partition into Voronou parallelohedra and the Delone partition for a lattice $\Lambda$ are dual to each other. Every face $F^{k}$ of the Voronou partition corresponds to a $(d-k)$-face $D^{d-k}\left(F^{k}\right)$ of the Delone partition, and vice versa; furthermore, $\operatorname{aff}\left(F^{k}\right) \perp \operatorname{aff}\left(D^{d-k}\left(F^{k}\right)\right)$.

Theorem 4.4 implies the following.

Proposition 4.1. The vertices of the Delone partition lie at the centres of the Voronor parallelohedra, and the vertices of the parallelohedra lie at the centres of the spheres circumscribed about the Delone polyhedra. If a parallelohedron is primitive, then the Delone partition is simplicial (the Delone triangulation), and if the parallelohedron is primitive at a $k$-face, then the corresponding $(d-k)$-face is a simplex.

\section{$\S 5$. The Uniqueness of a Voronou Parallelohedron}

As already mentioned, the following uniqueness theorem was proved in [11.

Theorem 5.1 (Michel, Ryshkov and Senechal). For every primitive parallelohedron $P$ there exists a unique affinely equivalent Voronou parallelohedron, up to similarity.

This theorem was strengthened significantly in [23]. Indeed, a parallelohedron $P$ is said to be semiprimitive if any two of its hyperfaces can be connected by a path passing from hyperface to hyperface only via nonstandard $(d-2)$-dimensional faces. In the case of a primitive parallelohedron $P$ there are no standard faces of codimension 2 , and so this condition is satisfied because its boundary $\partial P$ is a strongly connected $(d-1)$-complex. Hence a primitive parallelohedron is also semiprimitive. Furthermore, the parallelohedra mentioned in Zhitomirskiı's theorem, that is, parallelohedra that are primitive at all $(d-2)$-dimensional faces, also have no standard faces of codimension 2 and are therefore semiprimitive. In three-dimensional space, apart from the unique primitive parallelohedron - the truncated octahedron - there are also two semiprimitive parallelohedra: the elongated dodecahedron and rhombic dodecahedron.

\footnotetext{
${ }^{2}$ Voronor and Delone called this partition an L-partition; an amusing story about the origin of the term Delone partition can be found in [22].
} 
Theorem 5.2 (Dolbilin, Itoh and Nara [23]). For any semiprimitive parallelohedron $P$ there exists at most one Voronou parallelohedron (up to similarity) that is affinely equivalent to $P$.

Here we say "at most one" because, by contrast with those subclasses of parallelohedra for which the existence of an affinely equivalent Voronor parallelohedron is already established, the analogous problem for the class of semiprimitive parallelohedra as a whole remains unsolved. Below we present a significantly improved proof of Theorem 5.2.

Proof. First we prove the theorem for the two-dimensional case.

Lemma 5.1. For every convex centrally symmetric hexagon there exists a unique affinely equivalent hexagon inscribed in a unit circle.

Thus, let $P$ be a centrally symmetric hexagon. It is easy to see that such a convex hexagon is inscribed in an ellipse. We transform the circumscribed ellipse into a unit circle by a suitable affine transformation $\varphi$. Let $P_{V}=\varphi(P)$. We say that a hexagon inscribed in a unit circle is normalized. For a centrally symmetric normalized hexagon $P_{V}$ we can distinguish three central angles, $\alpha, \beta, \gamma$, based on its sides. We can assume without loss of generality that these angles satisfy the following inequalities:

$$
\alpha \leq \beta \leq \gamma, \quad \alpha+\beta+\gamma=\pi .
$$

Obviously, two normalized hexagons $H(\alpha, \beta, \gamma)$ and $H\left(\alpha^{\prime}, \beta^{\prime}, \gamma^{\prime}\right)$ are affinely equivalent to each other if and only if they are isometric: $\left(\alpha=\alpha^{\prime}, \beta=\beta^{\prime}, \gamma=\gamma^{\prime}\right)$.

Thus, any centrally symmetric hexagon $H$ is affinely equivalent to a unique normalized hexagon $H_{V}$, up to isometry. Since a normalized hexagon is inscribed in a circle, it is easy to see that for the lattice of centres of parallel copies of such a hexagon in a partition, the normalized hexagon is a Vorono $\breve{1}$ domain and therefore is a (two-dimensional) Vorono parallelohedron. The lemma and theorem for the case $d=2$ are proved.

We now consider a semiprimitive parallelohedron $P$, and let $P_{V}$ and $P_{V}^{\prime}$ be two Voronoŭ parallelohedra affinely equivalent to the parallelohedron $P$, and therefore equivalent to each other. We claim that two mutually affinely equivalent semiprimitive Voronol parallelohedra $P_{V}$ and $P_{V}^{\prime}$ are similar.

Consider a nonstandard face $F_{1}^{d-2}$ of codimension 2 in $P_{V}$ (if there were no such faces the complex $Q$ would not be strongly connected). The belt $B\left(F_{1}^{d-2}\right)$ consists of six hyperfaces. The corresponding facet vectors of this belt lie in one 2 -plane $\Pi_{1}$. Every facet vector in a Voronol parallelohedron is orthogonal to the corresponding hyperface, and hence it is also orthogonal to any of its proper subfaces. Hence the plane $\Pi_{1}$ is orthogonal to the $(d-2)$-face $F_{1}^{d-2}$.

Let the hexagon $H_{1}$ be the projection $p_{1}\left(P_{V}\right)$ of the parallelohedron $P_{V}$ along the $(d-2)$-plane of the face $F_{1}^{d-2}$ onto the orthogonal 2-plane $\Pi_{1}$. (Note that the intersection $P_{V} \cap \Pi_{1}$, generally speaking, does not coincide with $H_{1}$.) Since the facet vectors of the belt belong to the plane $\Pi_{1}$, under this projection they remain fixed (are projected to themselves). The hexagon $H_{1}$ has the following properties:

1) The hexagon is centrally symmetric.

2) Since the facet vectors in any parallelohedron pass through the centres of hyperfaces, the facet vectors of the belt under consideration pass through the midpoints of the sides of $H_{1}$.

3) Since the facet vectors in the Voronol parallelohedron $P_{V}$ are orthogonal to the hyperfaces, the facet vectors of the belt under consideration are perpendicular to the sides of the hexagon $H_{1}$, which lie in the planes of hyperfaces of this belt.

4) It follows from 1)-3) that the hexagon $H_{1}$ is inscribed in a circle of some radius. 
A similar conclusion can also be drawn about the corresponding hexagon $H_{1}^{\prime}$ in the parallelohedron $P_{V}^{\prime}$ : the hexagon $H_{1}^{\prime}$ is also inscribed in a circle of some, generally speaking, different radius.

Thus, if the parallelohedra $P_{V}$ and $P_{V}^{\prime}$ are affinely equivalent, $\varphi\left(P_{V}\right)=P_{V}^{\prime}$, then the hexagons $H_{1}$ and $H_{1}^{\prime}$ are also affinely equivalent. By Lemma 5.1 the restriction $\left.\varphi\right|_{\Pi_{1}}$ of the affine transformation $\varphi$ to the plane $\Pi_{1}$ is a similarity transformation of the hexagon $H_{1}$ to $H_{1}^{\prime}$ with some coefficient $\mu$, possibly $\mu=1$.

We now consider the belt $B\left(F_{2}^{d-2}\right)$ generated by another nonstandard face $F_{2}^{d-2}$. Suppose that this belt intersects the first belt in a hyperface with facet vector $\boldsymbol{t}$, say, and consists of six hyperfaces. The six facet vectors of the new belt lie in a 2-plane $\Pi_{2}$. The projection $p_{2}(P)$ onto the plane $\Pi_{2}$ is a centrally symmetric hexagon $H_{2}$, for which the facet vectors are vectors starting at the centre of the hexagon, passing though the midpoints of the sides of the hexagon, and perpendicular to these sides. Hence the hexagon $\mathrm{H}_{2}$ is inscribed in a circle, the radius of which is, generally speaking, different from the radius of the circle circumscribed about $H_{1}$.

Similar arguments can be conducted for the corresponding hexagon $H_{2}^{\prime}$ in the parallelohedron $P_{V}^{\prime}$. The restriction $\left.\varphi\right|_{\Pi_{2}}$ of the affine transformation $\varphi$ to the plane $\Pi_{2}$ is a similarity. We point out that both restrictions $\left.\varphi\right|_{\Pi_{1}}$ and $\left.\varphi\right|_{\Pi_{2}}$ take one and the same facet vector $\boldsymbol{t}$ in the parallelohedron $P_{V}$ to the corresponding facet vector $\boldsymbol{t}^{\prime}$ in the parallelohedron $P_{V}^{\prime}$. Hence both restrictions are similarity transformations with the same coefficient $\mu$.

Since the parallelohedron $P_{V}$ is semiprimitive, it is possible to pass from any of its hyperfaces to any other hyperface via nonstandard $(d-2)$-faces over a chain of belts $B_{1}, B_{2}, \ldots, B_{k}$ in which every two adjacent belts have a common hyperface (and the one antipodal to it). The corresponding restriction $\left.\varphi\right|_{\Pi_{i}}$, where $\Pi_{i}$ is the 2-plane of facet vectors of the belt $B_{i}$, is a similarity transformation with one and the same coefficient $\mu$ for any $i=1,2, \ldots, k$.

Thus, the affine transformation $\varphi$ is such that for every facet vector $\boldsymbol{t}$ in the parallelohedron $P_{V}$ and its $\varphi$-image $\boldsymbol{t}^{\prime}:=\varphi(\boldsymbol{t})$ in the parallelohedron $P_{V}^{\prime}$; we have $\left|\boldsymbol{t}^{\prime}\right|=\mu|\boldsymbol{t}|$.

Since this relation between the length of a vector and of its image holds for all facet vectors, and the set of all facet vectors $\boldsymbol{t}$ of the parallelohedron $P$ generates a $d$-dimensional space, the affine transformation $\varphi$ such that $\varphi\left(P_{V}\right)=P_{V}^{\prime}$ is a similarity transformation with coefficient $\mu$.

Corollary. If $P_{V}$ and $P_{V}^{\prime}$ are combinatorially equivalent semiprimitive Voronou parallelohedra, then they are either similar to each other (in particular, congruent) or they are not affinely equivalent.

\section{$\S 6$. Three unsolved problems}

Proving (or refuting) Voronol's conjecture on affine equivalence of any parallelohedron to some Voronol parallelohedron remains the central problem in the theory of parallelohedra. We now state several more questions, which are not as famous as the main Voronor problem. Note that Problem 3 (the 'dimension conjecture') is a significantly weakened version of Voronol's conjecture: an affirmative solution of the main conjecture would imply an affirmative solution of the dimension conjecture.

Problem 1 (Voronol). Let $P$ be a $d$-dimensional parallelohedron and let $\mathscr{F}$ be the set of all facet vectors (that is, vectors connecting the centre $O$ of the parallelohedron $P$ with the centres $O_{i}$ of the parallelohedra $P_{i}$ adjacent to $P$ over hyperfaces). Let $\Lambda$ be the point lattice generated by the set $\mathscr{F}$. Prove (or refute) that $\mathcal{F}$ always contains a basis of the lattice $\Lambda$. 
Let $T$ be a nonnormal partition of the space $\mathbb{E}^{d}$ into parallel and mutually congruent cells (nonnormal d-parallelohedra). It is known that a nonnormal parallelohedron also admits a normal partition, that is, it is a normal parallelohedron (see, for example, 9], [21]). We say that cells $P$ and $P^{\prime}$ are hyperneighbours if their intersection $P \cap P^{\prime}$ is a $(d-1)$-polyhedron.

Problem 2. Prove or refute the following assertion: any parallelohedron $P$ in the partition $T$ has at most $2\left(2^{d}-1\right)$ hyperneighbours.

Note that if the partition $T$ is normal, then the assertion is true (see Theorem 1.4).

Problem 3 (Dimension conjecture). Let $O_{1}, \ldots, O_{p}$ be the centres of all the parallelohedra of a normal partition that meet at a $k$-dimensional face $F^{k}$. Prove that

$$
\operatorname{dim} \operatorname{conv}\left(\left\{O_{1}, \ldots, O_{p}\right\}\right)=d-k .
$$

It is known that for any partition into Voronol parallelohedra the convex hull of the centres, $\operatorname{conv}\left(\left\{O_{1}, \ldots, O_{p}\right\}\right)$, is a $(d-k)$-dimensional face of the dual Delone partition. Thus, if Voronol's conjecture on affine equivalence holds, this would also imply the validity of the dimension conjecture. Correspondingly, a negative solution of the dimension conjecture would imply a refutation of Voronol's conjecture.

The author expresses his deep gratitude to Mikhail Ivanovich Shtogrin, who read the manuscript through carefully and was instrumental in improving the text.

\section{REFERENCES}

[1] E. S. Fëdorov, Basics of polytopes, St.-Petersburg, 1885; Moscow, 1953.

[2] H. Minkowski, Allgemeine Lehrsätze über die konvexen Polyeder, Nach. Ges. Wiss. Göttingen, 1897, 198-219.

[3] G. Voronoï, Nouvelles applications des paramètres continus à la théorie des formes quadratiques, II Mémoire: Recherches sur les paralléloédres primitifs, J. Reine Angew. Math. 134 (1908), $198-287$.

[4] B. N. Delaunay, Sur la partition regulière de l'espace à 4 dimensions. I, II, Izv. Akad. Nauk SSSR Ser. Mat. (1929), no. 1, 79-110; no. 2, 147-164.

[5] B. N. Delone, Geometry of positive quadratic forms, Uspekhi Mat. Nauk 3 (1937), 16-62; 4 (1938), 102-164. (Russian)

[6] O. K. Zitomirskij, Verschärfung eines Satzes von Woronoi, Zh. Leningrad. Fiz.-Mat. Ob-va 2 (1929), 131-151.

[7] B. A. Venkov, On a class of Euclidean polyhedra, Vestnik Leningrad Univ. Ser. Mat. Fiz. Khim. 9 (1954), 11-31. (Russian) MR0094790 (20:1302)

[8] A. D. Aleksandrov, Convex polyhedra, Gostekhizdat, Moscow-Leningrad, 1950; 2nd ed., in Selected works, 2, Nauka, Novosibirsk, 2007. (Russian) MR0040677 (12:732e)

[9] P. McMullen, Convex bodies which tile space by translation, Mathematika 27 (1980), 113-121. MR.582003(82c:52016)

[10] S. S. Ryshkov and E. P. Baranovskiǔ, $C$-types of $n$-dimensional lattices and five-dimensional primitive parallelohedra (with an application to the theory of coverings), Trudy Mat. Inst. Steklov. 137 (1976), 3-131. (Russian) MR0469874 (57:9655)

[11] L. Michel, S. S. Ryshkov and M. Senechal, An extension of Voronoï's theorem on primitive parallelotopes, Eur. J. Combin. 16 (1995), no. 1, 59-63. MR1317202 (95m:52028)

[12] M. I. Shtogrin, Regular Dirichlet-Voronő partitions for the second triclinic group, Trudy Mat. Inst. Steklov. 123 (1973), 3-128. (Russian) MR0370330 (51:6557)

[13] P. Engel, The contraction types of parallelohedra in $E^{5}$, Acta Cryst. 56 (2000), 491-496. MR 1784709 (2001f:52046)

[14] R. Erdahl, Zonotopes, dicings, and Voronoi's conjecture on parallelohedra, Eur. J. Combin. 20 (1999), no. 6, 527-549. MR1703597 (2000d:52014)

[15] G. M. Ziegler, Lectures on Polytopes, Springer-Verlag, Berlin, 1995. MR1311028 (96a:52011)

[16] N. P. Dolbilin, The extension theorem, Discrete Math. 221 (2000), no. 1-3, 43-59. MR1778907 (2001e:52038)

[17] N. P. Dolbilin and V. S. Makarov, An extension theorem in the theory of regular tilings and its applications, Trudy Mat. Inst. Steklova 239 (2002), 146-169; English transl., Proc. Steklov Inst. Math. 239 (2002), no. 4, 136-158. MR1975141 (2004c:52032) 
[18] P. Engel and V. P. Grishukhin, There are exactly 222 L-types of primitive five-dimensional lattices, Eur. J. Combin. 23 (2002), 275-279. MR1908650(2003i:11090)

[19] A. Ordin, Proof of the Voronoi conjecture on parallelotopes in a new special case, Ph.D. Thesis, Queen's University, Kingston, 2005. MR2708491

[20] N. P. Dolbilin, Minkowski's theorems on parallelohedra and their generalisations, Uspekhi Mat. Nauk 62 (2007), no. 4, 157-158; English transl., Russian Math. Surveys 62 (2007), no. 4, 793-795. MR 2358747(2008j:52022)

[21] N. P. Dolbilin, Properties of faces of parallelohedra. Geometry, topology and mathematical physics, Trudy Mat. Inst. Steklova 266 (2009), 112-126; English transl., Proc. Steklov Inst. Math. 266 (2009), 105-119. MR2603263 (2011i:52007)

[22] N. P. Dolbilin, Boris Nikolaevich Delone: his life and work, Trudy Mat. Inst. Steklova 275 (2011), 7-21; English transl., Proc. Steklov Inst. Math. 275 (2011), no. 1, 1-14. MR2962968

[23] N. Dolbilin, J. Itoh, and C. Nara, Affine equivalent classes of parallelohedra, 9th Int. Conf. Computational geometry, graphs and applications, Springer, Heidelberg, 2011, 55-60. MR2927310

[24] A. Magazinov, An upper bound for a valence of a face in a parallelohedral tiling, Preprint, 2012, arXiv: 1201.1539

[25] A. Gavrilyuk, A. Garber and A. Magazinov, The Voronoi conjecture for several new classes of parallelohedra, in preparation, 2012.

Steklov Mathematical Institute of the Russian Academy of Sciences

E-mail address: dolbilin@mi.ras.ru

Translated by E. KHUKHRO

Originally published in Russian 